\title{
Nonequilibrium Phase Transition and Current Filaments in Extrinsic Semiconductors*
}

\author{
Thomas Christen \\ Institut für Theoretische Physik, Universität Basel, Klingelbergstrasse 82, CH-4056 Basel
}

Z. Naturforsch. 49a, $851-855$ (1994); received May 30, 1994

\begin{abstract}
A phenomenological model for charge transport in extrinsic semiconductors is presented. For shallow impurities, large saturated impact-ionization coefficient and sufficiently low lattice temperature, the current-field characteristic is S-shaped (SNDC) due to the interplay between impact ionization and scattering of carriers from acoustic phonons, ionized and neutral impurities. The present model provides and explanation of the experimentally observed disappearance of SNDC above a critical lattice temperature. In the region of negative differential conductivity where a current-controlled uniform state is unstable, current filaments are calculated numerically.

PACS numbers : $72.20 . \mathrm{Ht}, 05.70 . \mathrm{Ln}$
\end{abstract}

\section{Introduction}

In samples of extrinsic semiconductors the transition of strictly monotonous to S-shaped current-field characteristics with negative differential conductivity (NDC) provides a typical example of a nonequilibrium phase transition [1-3]. The most striking similarity with equilibrium phase transitions is that bistability exists only below a critical temperature of the heat bath connected to the semiconductor sample. This property is often modelled by an appropriately chosen functional dependence of certain model parameters (generation-recombination (g-r) rates, mobility etc.) on the lattice temperature. In this paper an alternative explanation is proposed, where the relevant dependence of the model on the lattice temperature is related to the strength of inelastic scattering of carriers from acoustic phonons.

In Section II the model is introduced and the equations of motion are motivated. The current-field characteristic of uniform steady states is discussed in Section III. A well-known concomitant of S-shaped NDC (SNDC) is the formation of current filaments under certain conditions (e.g. for a current-controlled NDC state) [1-6]. In Section IV numerical solutions of the basic partial differential equations are presented, confirming the occurrence of stationary current filaments.

\footnotetext{
* Paper presented at the 4th Annual Meeting of ENGADYN, Grenoble, October 11-14, 1993.
}

Reprint requests to Dr. T. Christen, Institut für Theoretische Physik, Universität Basel, Klingelbergstrasse 82, CH-4056 Basel/Schweiz.

\section{The Model}

Consider a bulk semiconductor doped with shallow single-level impurities (donors or acceptors) of density $N\left(\approx 10^{15} \mathrm{~cm}^{-3}\right)$. Purely extrinsic conduction is realized at sufficiently low lattice temperature $(T \approx 4 \mathrm{~K})$. The sample is connected to an external circuit such that the total current flow (in $e_{z}$-direction) plays the role of the control parameter. A state is characterized by the electric field $\boldsymbol{E}$, the density $n_{0}$ of carriers with charge $q$ and constant effective mass $m$ in the conduction band, the density $n_{\mathrm{d}}$ of occupied impurities, the mean drift velocity $\boldsymbol{u}$, and the mean carrier energy $W$. It is reasonable to assume that at low electric fields almost all carriers are bound in impurity states. This implies that the mobility of the few free carriers is determined by the scattering at neutral impurities. At higher fields, when the value of the mean carrier energy is of the order of the ionization energy $W_{\text {ion }}$ $(\approx 0.5 \mathrm{meV})$ of an impurity, the carrier density increases abruptly due to the onset of impact ionization. Additionally, the nature of elastic scattering changes from neutral to ionized impurity scattering. While elastic scattering leads to momentum relaxation, the dilute gas of acoustic phonons being responsible for inelastic scattering causes a slow relaxation of energy.

A model with the above-mentioned physical ingredients can be formulated in the framework of the relaxation-time approximation of a set of hydrodynamic equations where the hydrodynamic modes, $n_{0}$, $\boldsymbol{u}$ and $W$ are governed by the balance of particle number, momentum and energy, respectively $[5,7]$. Due to 
the symmetry-breaking and the disorder caused by impurities and phonons, the hydrodynamic longwavelength spectra have gaps associated with g-r processes, momentum and energy relaxation.

First, g-r kinetics is assumed to consist of single-carrier capture and impact ionization. Thermal ionization and Auger recombination, which are the inverse processes, are neglected. A characteristic time is defined by the recombination time $\tau_{n}$, which is assumed to be constant. In order to describe impact ionization, the impact-ionization coefficient, on the other hand, is assumed to be proportional to $\exp \left(-\alpha W_{\text {ion }} / W\right)$ with positive $\alpha$. This type of g-r kinetics implies a strictly monotonous $n_{0}(W)$ for uniform steady states, where $n_{\mathrm{d}}=N-n_{0}$ is valid.

Secondly, momentum relaxation is characterized by an effective relaxation rate $\gamma_{p} / \tau_{p}$, where $\tau_{p}$ is a characteristic time and $\gamma_{\mathrm{p}}\left(n_{\mathrm{d}}, W\right)$ takes into account elastic scattering processes. Thus, $\gamma_{\mathrm{p}}=c_{1} n_{\mathrm{d}}+c_{2}\left(N-n_{\mathrm{d}}\right)$, where the coefficients $c_{1,2}(W)$ describe the dependence on energy of neutral and ionized impurity scattering, respectively.

Thirdly, energy relaxation is characterized by an effective relaxation rate $\gamma_{w} / \tau_{w}$, where $\tau_{w}$ is a characteristic time and $\gamma_{\mathrm{w}}(W)$ describes how acoustic phonon scattering depends on energy. It is important to realize that $\tau_{\mathrm{w}}^{-1}$ can be interpreted roughly as a measure for the lattice temperature, since the number of inelastic scattering processes decreases if the density of phonons becomes smaller. In principle, the specific dependences of the relaxation rates on energy can be derived from scattering theory. In the following, power-law approximations will be used $[5,8]$.

By anticipating that $\boldsymbol{u}$ will not slow down critically, one concludes from the order of magnitude of the characteristic times $\left(\tau_{\mathrm{n}} \approx 10^{-8} \mathrm{~s}, \tau_{\mathrm{p}} \approx 10^{-11} \mathrm{~s}\right.$, $\tau_{\mathrm{w}} \approx 10^{-9} \mathrm{~s}$ ) [3], that $\boldsymbol{u}$ can be eliminated adiabatically. Further, $\tau_{\mathrm{pw}} \equiv \tau_{\mathrm{p}} / \tau_{\mathrm{w}} \ll 1$ leads to an effective heating of carriers such that the thermal energy $w$ equals the total energy $W$, i.e. $w \equiv W-m \boldsymbol{u}^{2} / 2 \approx W$. Since $\tau_{\mathrm{wn}} \equiv \tau_{\mathrm{w}} / \tau_{\mathrm{n}} \gtrsim 0.1$, the thermal energy $w$ of a carrier becomes a slow variable, hence its time evolution must be included in the transport equations.

In order to express the transport equations in dimensionless form, it is convenient to choose a characteristic energy $\hat{W}$ (e.g. the ionization energy of impurities) defining a characteristic velocity $\hat{u} \equiv \sqrt{2 \tau_{\mathrm{pw}} \hat{W} / m}$ and a carrier diffusion constant $D \equiv \tau_{\mathrm{n}} \hat{u}^{2}$. After rescaling $t \rightarrow \tau_{\mathrm{n}} t, \boldsymbol{x} \rightarrow \sqrt{\tau_{\mathrm{n}} D} \boldsymbol{x}, n_{0, \mathrm{~d}} \rightarrow N n_{0, \mathrm{~d}}, \boldsymbol{u} \rightarrow \hat{u} \boldsymbol{u}$, $w \rightarrow \hat{W} w$, and $\boldsymbol{E} \rightarrow(\hat{u} / \mu) \boldsymbol{E}$ with the characteristic mo- bility $\mu=q \tau_{\mathrm{p}} / m$, the transport equations read

$$
\begin{gathered}
\partial_{\mathrm{t}} n_{0}+\boldsymbol{\nabla} \cdot\left(n_{0} \boldsymbol{u}\right)=f \equiv a_{\mathrm{I}} n_{0} n_{\mathrm{d}}-n_{0}\left(1-n_{\mathrm{d}}\right) \\
\partial_{\mathrm{t}} w+(\boldsymbol{u} \cdot \boldsymbol{\nabla}) w-\frac{D_{\mathrm{w}}}{n_{0}} \boldsymbol{\nabla} \cdot\left(n_{0} \boldsymbol{\nabla} w\right)+\frac{2}{3} w \boldsymbol{\nabla} \cdot \boldsymbol{u}+\frac{f}{n_{0}} w \\
=-\left(\gamma_{\mathrm{w}} w+\tau_{\mathrm{wn}} w_{\text {ion }} a_{\mathrm{I}} n_{\mathrm{d}}-2 \gamma_{\mathrm{p}} \boldsymbol{u}^{2}\right) / \tau_{\mathrm{wn}}
\end{gathered}
$$

where

$$
\begin{aligned}
\boldsymbol{u} & =\left(\boldsymbol{E}-\frac{\tau_{\mathrm{wn}}}{3 n_{0}} \boldsymbol{\nabla}\left(n_{0} w\right)\right) / \gamma_{\mathrm{p}}, \\
\gamma_{\mathrm{w}} & =\sqrt{w} \\
\gamma_{\mathrm{p}} & =n_{\mathrm{d}}+\gamma_{\mathrm{p}}^{(i)} w^{-3 / 2}\left(1-n_{\mathrm{d}}\right), \\
a_{\mathrm{I}} & =a_{\mathrm{I}}^{0} \exp \left(-w_{\text {ion }} / w\right) .
\end{aligned}
$$

Here, $w_{\text {ion }} \equiv \alpha W_{\text {ion }} / \hat{W}$ and $a_{\mathrm{I}}^{0}$ corresponds to the saturated impact-ionization coefficient. The quantity $\gamma_{\mathrm{p}}^{(i)}$ measures the strength of ionized impurity scattering compared to neutral impurity scattering. Usually, $a_{\mathrm{I}}^{0}$, $\gamma_{\mathrm{p}}^{(i)} \ll 1$ hold. In (2), the dimensionless heat diffusion constant $D_{\mathrm{w}}$ has been introduced according to Fick's law. The second term in the bracket on the r.h.s. of (2) takes into account the energy loss needed in order to ionize an impurity. Because the ratio of the lattice temperature and the effective carrier temperature is very small, the former will be neglected. In (3) it is assumed that no external magnetic field is present, and the Lorentz force of the internal magnetic field $\boldsymbol{B}$ acting on the carriers is neglected.

In order to take into account the dynamics of the electric and the magnetic fields, the transport equations must be supplemented by Maxwell's equations. The equation of motion for $n_{\mathrm{d}}\left(\partial_{\mathrm{t}} n_{\mathrm{d}}=-f\right)$ does not appear since this variable is expressed in terms of $n_{0}$ and $\boldsymbol{E}$ with the help of Poissons's equation

$$
\tau_{\mathrm{mn}} \boldsymbol{\nabla} \cdot \boldsymbol{E}=n_{0}+n_{\mathrm{d}}-1,
$$

where $\tau_{\mathrm{mn}} \equiv \tau_{\mathrm{m}} / \tau_{\mathrm{n}}$ and $\tau_{\mathrm{m}}=\varepsilon_{0} \varepsilon_{\mathrm{r}} / q \mu N$ is the dielectric relaxation time. Introducing the dimensionless magnetic-flux diffusion-constant $D_{\mathrm{B}} \equiv c^{2} \tau_{\mathrm{m}} / D$, where $c \equiv\left(\mu_{0} \varepsilon_{0} \varepsilon_{\mathrm{r}}\right)^{-1 / 2}$ is the velocity of light, and rescaling the magnetic field by $\boldsymbol{B} \rightarrow \mu_{0} q N D \boldsymbol{B}$, Maxwell's equations can be written in the form

$$
\begin{aligned}
& \tau_{\mathrm{mn}} \partial_{\mathrm{t}} \boldsymbol{E}-\boldsymbol{\nabla} \times \boldsymbol{B}=-n_{0} \boldsymbol{u}, \\
& \partial_{\mathrm{t}} \boldsymbol{B}+D_{\mathrm{B}} \boldsymbol{\nabla} \times \boldsymbol{E}=0
\end{aligned}
$$

(where, of course, $\boldsymbol{\nabla} \cdot \boldsymbol{B}=0$ ). Usually, $\tau_{\mathrm{mn}} \ll 1$ and $D_{\mathrm{B}} \gg 1$ hold. Equations (8) and (9) describe the dielectric relaxation and the diffusion of magnetic flux, re- 
spectively. Note that $\boldsymbol{B}$ should not be eliminated adiabatically since one finds that at the SNDC instability modes of magnetic flux with long wavelengths slow down critically.

Finally, appropriate boundary conditions must be specified. In the sequel, only bulk instabilities will be considered, and for $n_{0}, w$ and $\boldsymbol{E}$ homogeneous Neumann boundary conditions are assumed. Current control is guaranteed by prescribing appropriately the values of $\boldsymbol{B}$ at the sample boundaries since the total current is proportional to the line integral $\oint \boldsymbol{B} \mathrm{d} s$ along a path enclosing the current.

\section{Current-Field Characteristic and SNDC}

The current-field characteristic of the uniform stationary solution of (1) and (2) can be parameterized by $w$ :

$$
\begin{aligned}
& E \equiv E_{\mathrm{z}}=\sqrt{\frac{\gamma_{\mathrm{p}}}{2}\left(\gamma_{\mathrm{w}} w+\tau_{\mathrm{wn}} w_{\mathrm{ion}} n_{0}\right),} \\
& J \equiv \frac{1}{L_{\mathrm{x}} L_{\mathrm{y}}} \int j_{\mathrm{z}} \mathrm{d} x \mathrm{~d} y=\frac{n_{0}}{\gamma_{\mathrm{p}}} E,
\end{aligned}
$$

where $J$ is defined as the average current density and is obviously proportional to the total current. The carrier density is given by $n_{0}=a_{\mathrm{I}} /\left(1+a_{\mathrm{I}}\right)$. Typical solutions $J(E)$ with and without SNDC are plotted in Figure 1. The boundary of the NDC region is indi-

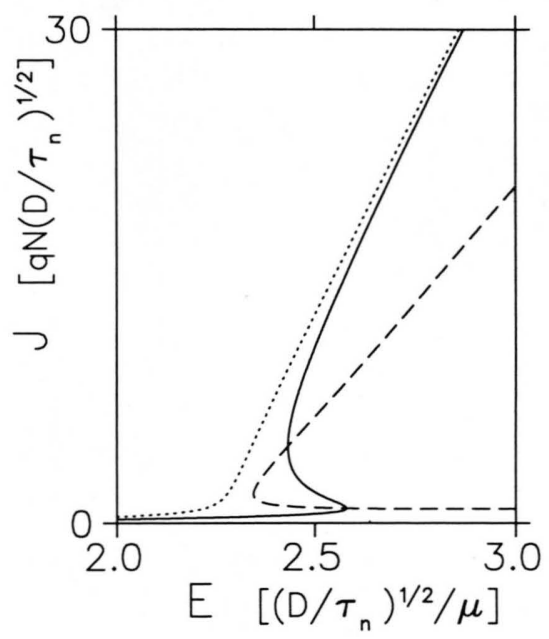

Fig. 1. $J-E$ characteristics; $a_{1}^{0}=30, \quad w_{\text {ion }}=5, \quad \gamma_{\mathrm{p}}^{(i)}=10$, $\tau_{\mathrm{wn}}=0.5$ (solid) and $\tau_{\mathrm{wn}}=0.1$ (dotted). The dashed curve indicates the spinodal line; SNDC exists above a critical $\tau_{\mathrm{wn}}$ $(\approx 0.22)$. cated by the (dashed) spinodal line. The region in the $a_{\mathrm{I}}^{0}-\ln \left(\gamma_{\mathrm{p}}^{(i)}\right)$ plane where SNDC occurs is shown in Figure 2.

The relevant mechanism producing SNDC is the rapid decrease of the effective mobility $\gamma_{\mathrm{p}}^{-1}$ caused by the onset of ionized impurity scattering at the onset of impact ionization. Closer inspection of (10) and (11) leads to the following results. First, SNDC exists only above a critical value of $\tau_{\mathrm{wn}}$, i.e. at weak phonon scattering. Hence, this model explains the phase-transition behaviour, i.e. the disappearance of SNDC above a critical lattice temperature. Secondly, in the present model SNDC exists for low ionization energies only, and therefore occurs favorably in samples with shallow impurities. Thirdly, as one expects, SNDC needs a sufficiently large saturated impact-ionization coefficient $a_{\mathrm{I}}^{0}$.

\section{Current Filaments}

Linear stability analysis shows that at the SNDC instability a soft transport mode couples to the magnetic flux mode, giving rise to a diffusion-type instability where the effective magnetic-flux diffusion-constant changes sign [1]. Diffusion-type instabilities often lead to the formation of spatially localized struc-

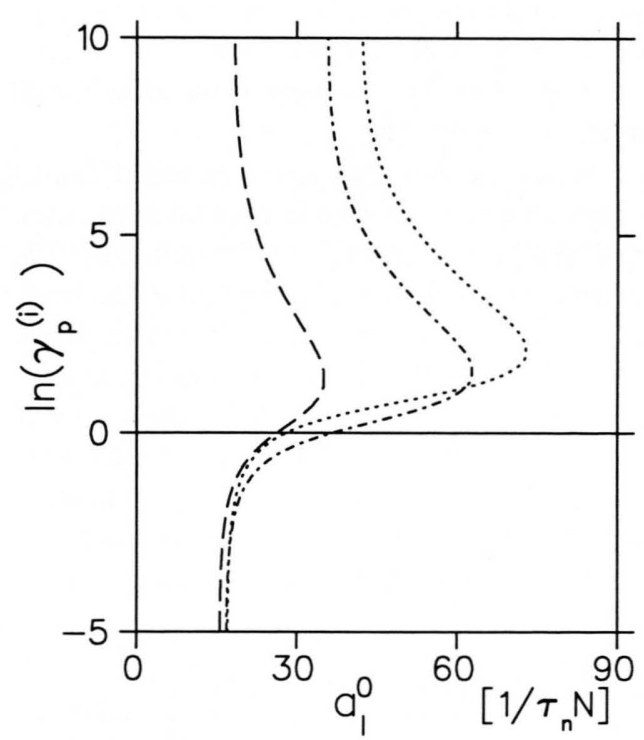

Fig. 2. Boundary between the regions of monotonous (left side) and S-shaped (right side) characteristics; $w_{\text {ion }}=5$, $\tau_{\mathrm{wn}}=0.2$ (dashed) and $\tau_{\mathrm{wn}}=0.1$ (dashed-dotted); $\tau_{\mathrm{wn}}=0.1$, $w_{\text {ion }}=7$ (dotted). 


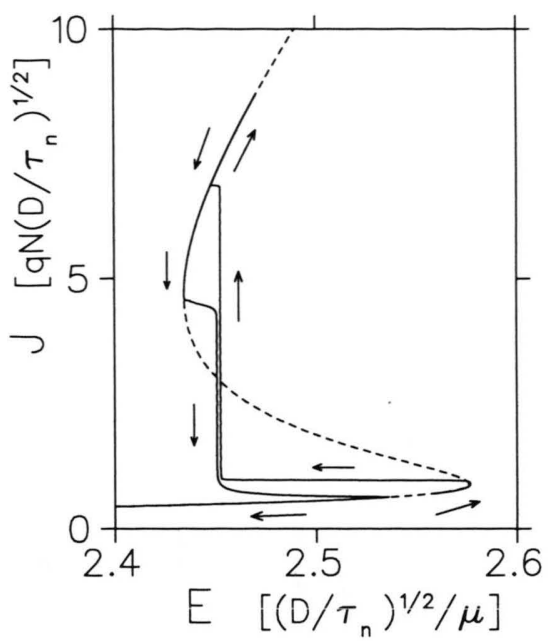

Fig. 3. Numerically obtained current-field characteristic (solid), characteristic of the uniform state (dashed). The arrows indicate the slow change of the control parameter $J$. Same parameter values as for the solid curve in Fig. 1, and $\tau_{\mathrm{mn}}=10^{-3}, D_{\mathrm{w}}=1, D_{\mathrm{B}}=10^{4}$. Instability points: $(E, J)_{\min }$ $=(2.576,0.897)$ and $(E, J)_{\max }=(2.435,4.666)$.

tures which are current filaments in the present case. In order to confirm the formation of localized states, the transport equations coupled to Maxwell's equations are solved numerically ${ }^{1}$. For the sake of simplicity, uniformity of the solution in direction of the current $\left(\boldsymbol{e}_{z}\right)$ and the case of a single transverse space dimension $\left(\boldsymbol{e}_{\mathrm{x}}\right)$ is assumed ${ }^{2}$.

Figure 3 shows the $J-E$ characteristic (solid curve) obtained from an adiabatic loop in control parameter space. The dashed curve belongs to the uniform solution (solid curve in Figure 1). The current $J$ has been increased slowly with velocity $\mathrm{d} J / d t=10^{-3}$ from $J=0.7$ to $J=8.7$, from where it has been decreased with velocity of equal amount. At the SNDC instability, a stationary current filament with finite amplitude forms. In Fig. 4 the nucleation of the filament at a boundary of the sample is shown. By increasing the current, the size of the filament grows at constant electric

1 IMSL-routine 'DMOLCH' (Method of lines).

2 The numerical (current-controlled) solution with two transverse dimensions and cylindrical symmetry can be obtained in the same way, using the radial coordinate only. In this case, the control parameter corresponds to the value of the azimuthal component $B_{\varphi}$ of the magnetic field at the sample boundary.

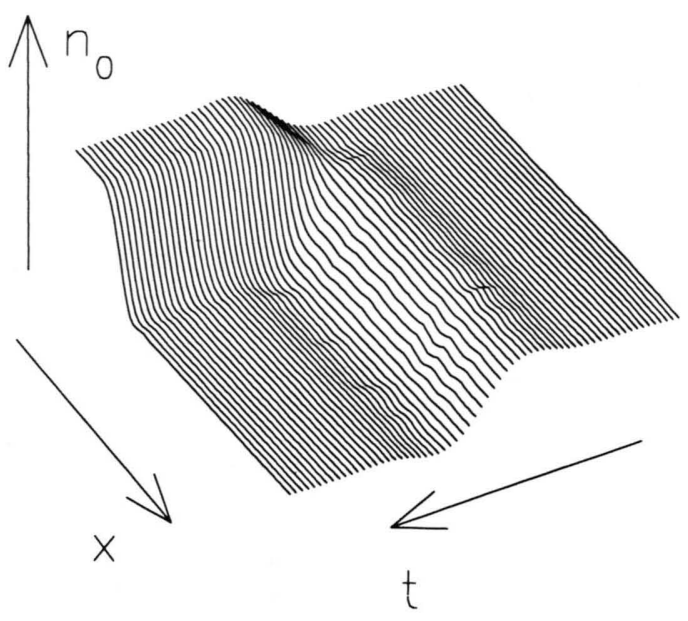

Fig. 4. Surface plot of the carrier density $n_{0}$ close to $J=0.897$ (see Fig. 3): a current filament forms at the sample boundary.

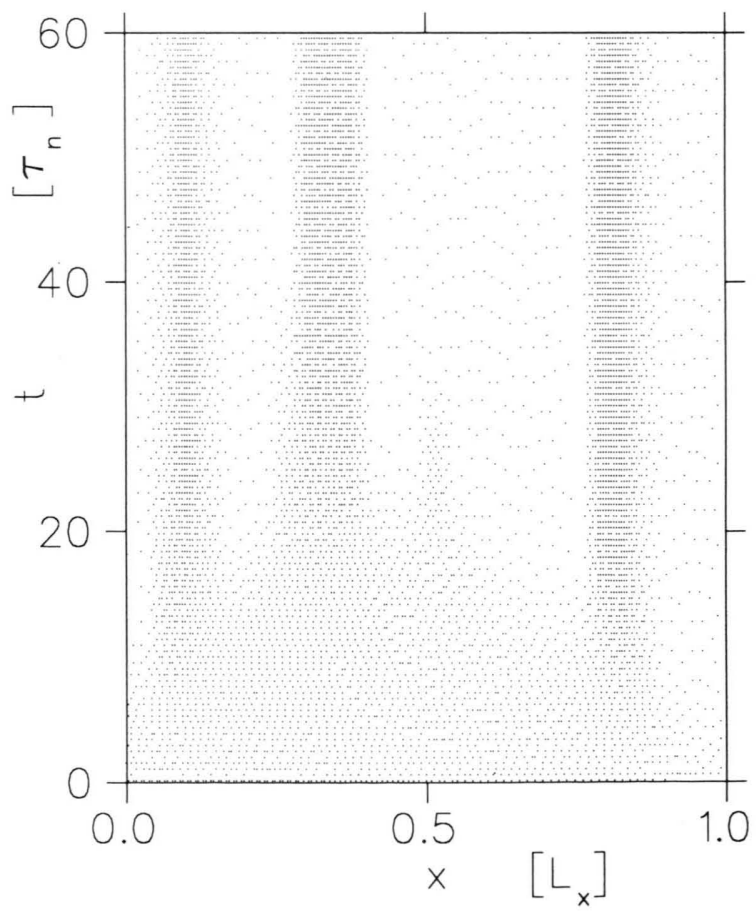

Fig. 5. Carrier-temperature density-plot of a quench into the unstable region $(J=2.0)$. A state with three filaments emerges; high density corresponds to hot carrier gas inside the filaments (same parameter values as in Figure 3). 
field until the high current-density state fills the whole bulk. The hysteresis of the filamentary branch near the instability points comes from the subcritical nature of the bifurcation of the localized solutions. Finally, it is mentioned that the small finite gap between the rising and the falling filamentary branch and the small wiggles of the characteristic are caused by the pinning of the filament wall at the mesh points used in the numerical simulation.

A multi-filament state can be obtained by preparing a strongly unstable uniform bulk state, where the initial exponential growth of small localized fluctuations is very fast compared to the relaxation to the stable nonuniform state. In Fig. 5 a space-time density-plot of the carrier temperature belonging to such a quench is shown. Hot regions corresponds to high carrierdensity filaments. This figure illustrates the diffusiontype instability associated with a negative diffusion constant: a uniformly distributed density contracts and concentrates in certain regions of the sample. The saturation of the contraction occurs on a scale determined by the energy-diffusion length and the Debye length. Note that a multi-filament state is actually unstable due to interaction between current filaments. In the one-dimensional case, however, the interaction force turns out to be exponentially small with respect to the distance of the filament walls, and the multi-filament state appears to be stationary due to the 'numerical pinning' of the walls at the mesh points.

[1] E. Schöll, Nonequilibrium Phase Transitions in Semiconductors, Springer Series in Synergetics, Vol. 35, Berlin 1987.

[2] B. Röhricht, R. P. Huebener, J. Parisi, and M. Weise, Phys. Rev. Lett. 61, 2600 (1988).

[3] U. Rau, Räumliche und zeitliche Strukturbildung beim nichtlinearen Ladungstransport in Halbleitern, Thesis, Universität Tübingen 1991.

[4] G. Hüpper, K. Pyragas, and E. Schöll, Phys. Rev. B 47, 15515 (1993).

[5] V. L. Bonch-Bruevich, I. P. Zvyagin, and A. G. Mironov, Domain Electrical Instabilities in Semiconductors, Consultants Bureau, New York 1975.

\section{Summary}

A specific interplay of impact ionization, scattering of carriers from impurities and acoustic phonons is proposed as a mechanism producing SNDC in extrinsic semiconductors. In the framework of the relaxation-time approximation, a phenomenological model is formulated which shows qualitatively the vanishing of SNDC above a critical lattice temperature as reported in [2]. With the help of one-dimensional numerical solutions of the basic equations of motion, the formation of current-controlled stationary current filaments and the current-field characteristic associated with filaments is investigated qualitatively.

It should be mentioned that additionally a longitudinal instability at finite wavelength and frequency can occur in semiconductors of the type considered in this paper [9]. The explanation of the frequently observed complex spatio-temporal dynamics [6] should include both transverse and longitudinal instabilities and their interaction.

\section{Acknowledgements}

The author wishes to thank H. Thomas, F. J. Elmer, and the members of ENGADYN for many helpful discussions. This work has been supported by the Swiss National Science Foundation.

[6] J. Peinke et al., Spatio-Temporal Correlations in Semiconductors, in: Spontaneous Formation of Space-Time Structures and Criticality (T. Riste and D. Sherrington, eds.), Nato ASI C 349 (1991).

[7] E. Schöll, Current Instabilities in Semiconductors: Mechanisms and Self-Organized Structures, in: Nonlinear Dynamics in Solids (H. Thomas, ed.), Springer-Verlag, Berlin 1992.

[8] K. Seeger, Semiconductor Physics, Springer-Verlag, Berlin 1973.

[9] T. Christen, Phys. Rev. B 49, 16423 (1994). 